\title{
9. Die Rolle der KI beim automatisierten Fahren
}

\author{
Marcel Kappel, Edgar Krune, Martin Waldburger, Benjamin Wilsch
}

Mobilität wird künftig von einer zunehmenden Elektrifizierung, Automatisierung und Vernetzung der Transportmittel geprägt sein. Während es sich bei der Elektrifizierung im Kern um die Ablösung einer bestehenden Antriebstechnologie handelt, erweitern Automatisierung und Vernetzung bestehende Mobilitätskonzepte und verursachen somit einen grundlegenden Wandel in der Mobilität. Für die automatisierte Steuerung von Transportfahrzeugen, insbesondere von Autos im Straßenverkehr, ist KI eine unersetzliche Schlüsseltechnologie und bietet im Zusammenspiel mit der Vernetzung zahlreiche Möglichkeiten verkehrsträgerübergreifender Koordination. Allerdings ist die Übergabe der Fahrverantwortung an die KI bedenklich für die Sicherheit, sodass sich für die Lernphase entsprechend hohe Anforderungen ergeben. Die Nutzung von KI-Methoden zur Gestaltung der Mobilität verspricht jedoch einen hohen Sicherheits-, Komfort- und Effizienzgewinn und bietet damit die Motivation für intensive Forschungs- und Entwicklungsaktivitäten.

Trotz der potenziellen Vorteile und der hohen Relevanz von KI in Bezug auf eine intelligente Mobilität sind die Effekte für Endnutzer heute noch nicht wahrnehmbar, da sich in diesem Bereich noch keine wirkliche Breitenwirkung entfalten konnte..$^{54}$ Jedoch wurden im Laufe des vergangenen Jahrzehnts insbesondere durch die Entwicklung leistungsfähiger und anwendungsorientierter Hardware die technischen Voraussetzungen für den KI-Einsatz im Fahrzeug geschaffen. Deren erfolgreicher Einsatz in der Praxis erfordert allerdings große Datenmengen (Big Data) als Grundlage.

Aufgrund der zunehmenden Vernetzung der Gesellschaft und der Infrastrukturen sind derzeit jedoch die Menge, Vielfalt und Verfügbarkeit dieser Daten gewachsen, und es wurden erste Forschungsentwicklungen initiiert, die sich aber vorrangig auf Leuchtturmprojekte für intelligente Mobilität konzentrierten. Zudem lassen sich zahlreiche innovative Forschungsarbeiten identifizieren, die auf europäischer, nationaler und regionaler Ebene über gezielte Fördermaßnahmen (z. B. Modernitätsfonds,

54 Tatsächlich gibt es aber durchaus technologische Bereiche, in denen KI mittlerweile eine Breitenwirkung erzeugt. Zu nennen ist hier beispielsweise die Analyse von Bild- und Videoinhalten mittels tiefem Lernen (Deep Learning, DL - z. B. "Google Photo" und "Microsoft Azure") und die Spracherkennung bei Assistenzsystemen. 
Deutscher Mobilitätspreis etc.) Unterstützung erhalten. Auch sind erste KI-Bausteine in Anwendungen mit Mobilitätsbezug erkennbar, zum Beispiel digitale Assistenten oder eine dynamische, multimodale Navigationsunterstützung.

In der Summe spielt KI in heutigen Mobilitätsanwendungen jedoch noch keine wesentliche Rolle. Weder gibt es eine (wirkliche) "Smart City", in der Verkehrsflüsse intelligent und nachhaltig gesteuert und alle Verkehrsträger sowohl untereinander als auch mit der Infrastruktur vernetzt wären. Noch existiert eine überzeugende Sprachsteuerung, mit der es möglich wäre, ein komplexes Gespräch über längere Zeit zu führen, wie es für eine intuitiv nutzbare Fahrer-Fahrzeug-Kommunikation in beide Richtungen notwendig wäre.

Möglicherweise lässt sich der aktuell geringe KI-Einsatz in der Mobilität damit erklären, dass auf Daten basierende Lösungen von Mobilitätsproblemen besonders aufwendig sind, denn Mobilität und Verkehr sind ein überaus komplexes Handlungsfeld, das einen wachsamen Fahrer in schnell wechselnden Situationen erfordert. Die Herausforderung für KI besteht also in der Steuerung hoch dynamischer, komplexer Systeme mit hohem Heterogenitätsgrad. Bevor KI in diesem Umfeld sinnvoll eingesetzt werden kann, muss zunächst eine geeignete und ausreichende Datenlage geschaffen werden. Die datenmäßige Erfassung, Modellierung und intelligente Analyse solcher Systeme ist allerdings höchst anspruchsvoll; eine Digitalisierung der Infrastruktur von Städten sowie von Fahrzeugen und deren Vernetzung untereinander sind Vorbedingungen für den Erfolg von KI in der Mobilität. Zwar werden Digitalisierung und Vernetzung derzeit mit Hochdruck verfolgt - wenn auch für die Sensorausstattung der zu vernetzenden Infrastruktur ein großer Aufwand vonnöten ist -, allerdings ist bislang noch kein KI-Einsatz größeren Umfangs erkennbar. Ein Hemmnis ist sicherlich die mangelnde Datenverfügbarkeit, weil die datenerhebenden und datenhaltenden Akteure so unterschiedlich sind. Und da zumal personenbezogene Datensätze sowie Sensorrohdaten von hohem Wert sind, werden sie nicht ohne weiteres Dritten zur Verfügung gestellt.

Wenn diese Hürden aber genommen werden können, wenn also eine umfassende und geeignete Datenverfügbarkeit gewährleistet ist, ist die Grundlage dafür gelegt, dass sich das enorme Potenzial für den Einsatz von KI in zukünftigen Mobilitätsanwendungen entfalten kann. Dann wird die KI einen wesentlichen Beitrag bei der Optimierung von Verkehrsflüssen leisten. Dies gilt gleichermaßen für den Warenund Personenverkehr. Beispielsweise können mittels multimodaler Verkettung Personenströme besser aufeinander abgestimmt werden und in der Konsequenz die vorhandene Verkehrsinfrastruktur entlasten. Auch können durch geschicktes Lenken der Personen- oder Warenverkehre bisher ungenutzte Freikapazitäten erschlossen werden. Beispielsweise lassen sich Grünphasen von Ampeln an das Verkehrsaufkommen anpassen. Von großer Bedeutung ist zudem das Potenzial von Kl, die 
Mobilität von morgen sicherer zu gestalten. So wird eine Projektion der möglichen Bewegungspfade von Verkehrsteilnehmern an einer Kreuzung die Risikoeinschätzung von Kollisionen oder sonstigen Gefahrensituation im Voraus ermöglichen - in Kombination mit einer Warnmöglichkeit ließen sich so Unfälle vermeiden. Aber auch auf dem Weg zu einer umweltfreundlicheren Mobilität kann der Einsatz von KI viel leisten. Einerseits lässt sich eine Reduktion von Emissionen mittels der bereits erwähnten Verkehrsflussoptimierungseffekte herbeiführen, andererseits können auch in weniger offensichtlichen Bereichen ressourcenschonende Lösungen gefunden werden, beispielsweise im Bereich der Elektromobilität, indem über eine intelligente Ladezyklussteuerung die Lebensdauer von Batterien bei gleichzeitig optimierter Reichweite erhöht werden kann. Schließlich birgt KI nicht nur das Potenzial zur Verbesserung bestehender Mobilitätsformen. Für neue Mobilitätsformen wie autonome Flugtaxis oder Logistik-Drohnen wird der Einsatz von Kl eine entscheidende Rolle spielen.

\section{Fokus der Forschung und Entwicklung liegt auf dem automatisierten Fahren}

Die bisher erwähnten Anwendungsfelder verdeutlichen das weitreichende Potenzial von KI für die Mobilität der Zukunft. Trotz der vielfältigen Anwendungsmöglichkeiten lässt sich bereits heute erahnen, in welchen Bereichen das primäre Augenmerk der Forschung und Entwicklung liegen wird. Ein solcher Bereich ist das automatisierte Fahren, was im Folgenden im Sinne eines Beispiels von besonderer Bedeutung näher betrachtet wird. Angesichts eines schon bestehenden hohen Automatisierungsgrades im Flug- und Bahnverkehr, wird in Zukunft die größte - potenziell disruptive Veränderung in der Mobilität das automatisierte Fahren sein. Hier schafft KI überhaupt erst die technologischen Voraussetzungen für die Automatisierung. Im Zukunftsbild einer multimodalen Mobilität, welche die Vorteile der Automatisierung, Elektrifizierung und Vernetzung sowie der geteilten Nutzung vereint, kann KI die Nutzung von Synergiepotenzialen sowie die verkehrsträgerübergreifende Optimierung der Reiserouten gewährleisten, wirklich interessant wird ihr Einsatz jedoch dort, wo sie Funktionen übernimmt, die bisher nur von Menschen ausgeübt werden konnten. Die technologischen Durchbrüche in einzelnen Bereichen lassen sich anhand des automatisierten Fahrens veranschaulichen und auf andere Segmente der Mobilität der Zukunft übertragen. Im Folgenden wird daher der Fokus auf die wesentlichen Anwendungen der KI für das automatisierte Fahren gelegt, die sich wie in Abbildung 9.1 dargestellt zusammenfassen lassen: 


\section{Automatisiertes Fahren}

Semantische Umfelderkennung

Fahrzeugsteuerung (Verhaltensplanung und -regelung)

\section{Fahrer-Fahrzeug-Interaktion}

Spracherkennung

Fahrer-/Passagierzustandserkennung

\section{Verkehrsflussoptimierung \\ Flottenmanagement \\ Vorbeugende Wartung}

Abbildung 9.1: Überblick der Anwendungsbereiche der KI für das automatisierte Fahren.

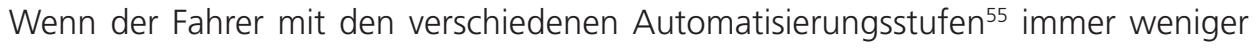
Aufgaben der Fahrzeugsteuerung übernimmt, erhält die $\mathrm{KI}$ ein weiteres Anwendungsfeld, die Fahrer-Fahrzeug-Interaktion. Und bei zunehmender Vernetzung der

55 Um die verschiedenen Abstufungen des assistierten Fahrens bis hin zum vollautomatisierten Fahren besser zu beschreiben und zusätzlich auch eine klare rechtliche Abgrenzung zu definieren, wurden die fünf Stufen (bzw. sechs bei Berücksichtigung einer nullten Stufe für die Fahrzeuge ohne jegliche Assistenzfunktion) des automatisierten Fahren eingeführt (National Highway Traffic Safety Administration). Hierbei gilt: Je höher die Stufe, desto höher der Automatisierungsgrad. 
Verkehrsmittel sowie der Infrastrukturen kann KI darüber hinaus den Verkehrsfluss optimieren.

Die genauere Betrachtung des automatisierten Fahrens zeigt, dass KI hierbei zunächst genutzt wird, um das Umfeld zu erkennen. In diesem Entwicklungsbereich wurde die Zuverlässigkeit der KI z. B. im Rahmen des ImageNet-Wettbewerbs kontinuierlich verbessert und übertraf zuletzt sogar menschliche Fähigkeiten (Wu et al. 2015). Auch an einer KI-gestützten Fahrzeugsteuerung wird derzeit intensiv geforscht. Prototypen zeigen immer bessere und auch schon zuverlässige Leistungen in komplexen Verkehrssituationen, wie z. B. in urbanen oder verschneiten Gebieten. Wichtiges Forschungsziel ist zum Beispiel das Erlernen einer intuitiven Fahrstrategie. Dass KI tatsächlich Intuition lernen kann, wurde bereits beispielsweise beim Go-Spielen deutlich (Silver et al. 2017). Bislang fokussierte sich die Forschung zum automatisierten Fahren allerdings darauf, prinzipielle Funktionsweisen aufzuzeigen und Leistungsgrenzen zu ermitteln. Für den Durchbruch des automatisierten Fahrens wären eine effiziente Verarbeitung der zahlreichen Sensordaten in Echtzeit und daher eine deutliche Optimierung der Hardware erforderlich. Dabei ist davon auszugehen, dass die innerhalb eines Fahrzeugs generierte Datenmenge ständig größer wird. In diesem Zusammenhang ist auch (speziell bei Elektrofahrzeugen) der zunehmende Stromverbrauch zu berücksichtigen ${ }^{56}$ (IEEE Spectrum 2018). Er erhöht sich durch die aus Sicherheitsgründen erforderliche Redundanz der Systeme und damit deren Kontrolle um ein Vielfaches.

Der Bedarf an leistungsfähiger und effizienter Hardware für KI-Anwendungen ist die Ursache, dass Autohersteller, Zulieferer und IT-Unternehmen entsprechend kooperieren und die spezifischen Forschungs- und Entwicklungsarbeiten weiter zunehmen. Die Autohersteller haben erkannt, dass sie mit dem vorhandenen Know-how in den Unternehmen und dem Ausbildungsstand der Belegschaften die Herausforderungen einer KI-Entwicklung für das automatisierte Fahren nicht erfolgreich werden bewältigen können. Über Akquisitionen, Investitionen und/oder Kollaborationen verschaffen sie sich deshalb gegenwärtig Zugang zu den Kompetenzen von auf KI spezialisierten Unternehmen, z. B. VW und Hyundai mit Aurora, Ford mit Argo Al.

\section{Auswirkungen des automatisierten Fahrens auf die Mobilität}

Das automatisierte Fahren eröffnet Möglichkeiten, die Mobilität der Menschen sowie den urbanen Raum einschneidend zu verändern. Es lassen sich drei Visionen anfüh-

56 Mittlerweile ist die Verwendung von KI auch beim intelligenten Batteriemanagement durchaus interessant. Jedoch ist der Effekt nicht groß genug, um den signifikanten Mehrbedarf in der Zukunft zu kompensieren, der durch die Automatisierung entsteht. 
ren: Erstens soll die Leistungsfähigkeit des Menschen erweitert werden, da automatisierte Fahrzeuge die Mobilität für alle (Minderjährige, Senioren und Menschen mit Behinderungen) erhöhen oder überhaupt erst ermöglichen. Die zweite Vision ist eine deutliche Reduzierung der Verkehrsunfälle. Dies ist ein zentrales Argument für die Einführung des automatisierten Fahrens, da mehr als 90 Prozent der jährlich 1,25 Millionen Verkehrstoten weltweit auf menschliches Versagen zurückzuführen sind (Smith 2013). Dies, so die Hoffnung, ließe sich in einem vollautomatisierten und vollständig vernetzten Verkehrssystem vermeiden. Auf eine Optimierung der Verkehrsflüsse zielt eine dritte Vision, es geht um die Entlastung des urbanen Verkehrs. Dies könne z. B. durch eine größere Attraktivität von (neuen) Alternativen zum privaten und personenbezogenen Fahrzeug erreicht werden. Die drei Vorstellungen, Mobilität durch $\mathrm{KI}$ zu verbessern, visualisiert Abbildung 9.2.

\section{Nutzerakzeptanz als Erfolgsfaktor für das automatisierte Fahren}

Der Durchbruch neuer Technologien hat neben seinen technischen oft auch soziale Aspekte. Bis das automatisierte Fahren eine alltägliche und selbstverständliche Angelegenheit für uns alle sein wird, müssen die Entwickler noch einige Hürden auf jeder dieser Ebenen überwinden. Zum Beispiel können die notwendigen Lernphasen für die unterschiedlichen $\mathrm{KI}$-Anwendungen, insbesondere wenn es um Sicherheit geht, nicht einfach im realen Verkehr stattfinden (siehe Einleitung zu Kapitel Technologie „Entwicklungswege zur KI"). Gleichwohl wird der KI-Algorithmus erst durch Lernvorgänge kontinuierlich besser und robuster. Sind hierfür ausreichend Vergleichsdaten in entsprechender Güte vorhanden, kann anhand dieser Datenbasis eine Phase der Anwendungsalgorithmen einsetzen, in der sie "angelernt" werden, z. B. kritische Situationen im Straßenverkehr zu erkennen und darauf zu reagieren. Oder sie lernen, mittels Spracherkennung intuitiv mit dem Fahrer zu interagieren, die Gefühlslage des Fahrers bzw. der Passagiere einzuschätzen, um aktiv darauf einzugehen.

Wenn auch eine KI-Anwendung im Laufe der Zeit immer zuverlässiger wird, ist in frühen Stadien mit einer hohen Fehlerquote zu rechnen. In den sicherheitsrelevanten Anwendungen der Mobilität ist dies jedoch inakzeptabel. Für das automatisierte Fahren können falsche Ergebnisse etwa beim Erkennen der Umgebung potenziell lebensbedrohlich sein und dürfen weder vom Hersteller noch vom Nutzer hingenommen werden.

Und das automatisierte Fahren wirft weitere Fragen auf, die derzeit ungeklärt sind, insbesondere ethischer Natur. Was soll geschehen, wenn das autonome Fahrzeug einen unausweichlichen Unfall detektiert und urteilen muss, ob Personen-, Tier-, oder Sachschäden vorzuziehen sind (Ethik-Kommission 2017)? Eine Implementierung derartiger Entscheidungen in einen Algorithmus möchte wohl kein Autohersteller und erst recht keine Ingenieurin und kein Ingenieur verantworten. Auch fehlt derzeit 


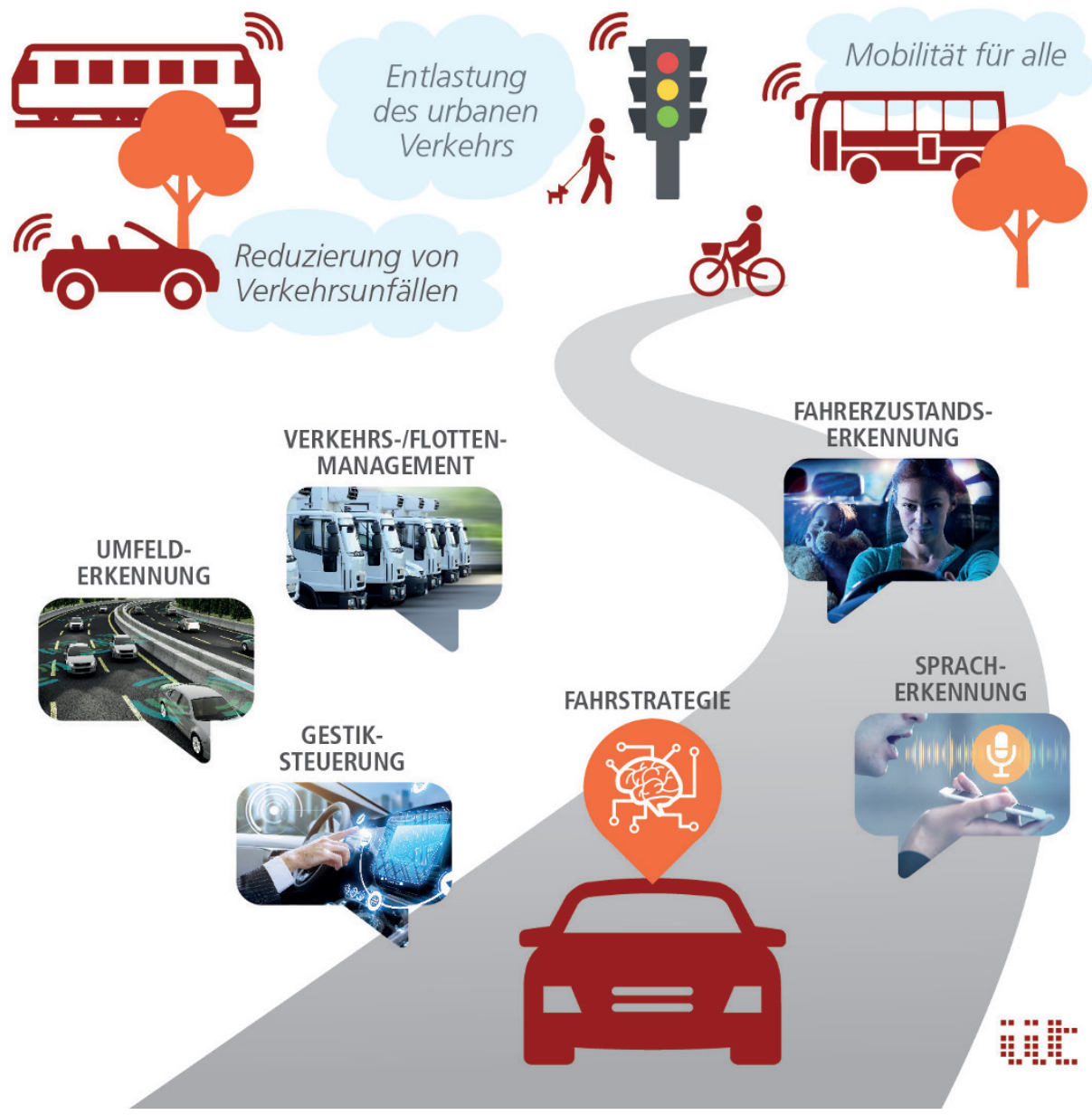

Abbildung 9.2: Überblick der Einsatzbereiche von Kl im Fahrzeug sowie der gesellschaftlichen Potenziale, die ein hochautomatisiertes und in die Stadt der Zukunft integriertes Fahrzeug erschließen kann (eigene Darstellung ${ }^{57}$ ).

eine Rechtsgrundlage, um Unfälle mit KI-Beteiligung zu bewerten. Es besteht das grundsätzliche Problem einer derzeit nicht möglichen Plausibilisierung von KI-Entscheidungen.

57 Bildnachweise: Thinkstock/metamorworks (Gestiksteuerung und Spracherkennung), Adobe Stock/folienfeuer (Umfelderkennung), LVDESIGN (Verkehrs-/Flotten-Management), jackfrog (Fahrerzustandserkennung) 


\section{Unterschiedliche Entwicklungspfade der Automatisierung durch KI}

Trotz umfangreichen Trainings ist jede durch $\mathrm{KI}$ getroffene Entscheidung mit Unsicherheiten behaftet - besonders dann, wenn die KI auf Daten außerhalb der Trainingsmenge angewandt wird. Aber ab wann ist ein KI-Assistenzsystem gut genug für die praktische Anwendung im Feld? Und wie kann man trotz Lernphase diese Technik insbesondere bei Anwendungen, welche die Sicherheit tangieren, in vorhandene Systeme etablieren?

Um eine trainierte KI zu verwenden, müssen also zunächst neue Validierungsmethoden und Sicherheitsmechanismen geschaffen werden, mit denen sich fehlerhafte KI-Entscheidungen auffangen lassen. Hierbei haben sich zwei Vorgehensweisen etabliert: Ein Ansatz ist die Einbindung der KI über die Teilautomatisierung des Fahrzeugs (z. B. Tesla, Audi, Daimler), wobei einzelne Fahrfunktionen in das Fahrzeugsystem schrittweise integriert werden (z. B. Update-Möglichkeit bei Tesla). Ein weiterer Ansatz (z. B. von Apple und der Alphabet-Tochter Waymo) sieht vor, ohne Zwischenschritt direkt hoch- und vollautomatisierte Lösungen zu entwickeln.

Die Teilautomatisierung hatsich als besonders geeignet für etablierteFahrzeughersteller herausgestellt, da hierbei auf bereits vorhandene Fahrassistenzsysteme aufgebaut werden kann und somit eine hoch- bis vollautomatisierte Lösung schrittweise über verschiedene Automatisierungsstufen angestrebt wird. Ist ein robustes Niveau der Fahrfunktion erreicht, kann der Fahrer das Assistenzsystem aktiv freischalten und nutzen. Da derzeit keine Validierungsmethoden zur Verfügung stehen, um die SicherheitdieserFahrfunktionenzugewährleisten, kann bei diesem Entwicklungsansatz auf den Fahrer als Beobachter, der in brenzligen Situationen unmittelbar eingreift, nicht verzichtet werden. Diese Überwachungsfunktion des Fahrers kann allerdings schließlich auch Vertrauen in $\mathrm{KI}$ aufbauen.

Im Kontrast dazu entwickeln die neu hinzugekommenen, softwarespezialisierten Akteure in der Automobilindustrie direkt vollständig selbstfahrende Fahrzeuge - zum Teil bereits ohne Lenkrad. Sie begründen dieses Vorgehen mit Erkenntnissen aus Studien, in denen die wechselnde Übergabe der Steuerung zwischen Mensch und Maschine untersucht und als zusätzliches Risiko identifiziert wurde.

Keiner dieser beiden Ansätze hat sich bislang als Königsweg herauskristallisieren können. Für beide Wege kann angenommen werden, dass sich in der Entwicklungsphase, d. h. im Mischverkehr aus nicht-, teil- und vollautomatisierten Fahrzeugen, Unfälle mit Personenschäden nicht ausschließen lassen, und sie sind auch schon aufgetreten. 


\section{Aufbau von Vertrauen in automatisierte Fahrfunktionen}

In der Diskussion um Nutzerakzeptanz gilt es, ungleich gelagerte Toleranzschwellen für menschliche und maschinelle Fehler zu berücksichtigen. Wie sensibel die Öffentlichkeit Sicherheit und deren Gewährleistung bzw. Nichtgewährleistung im Mischverkehr wahrnimmt, zeigt bereits der erste tödliche Unfall eines sich im automatisierten Modus befindlichen Fahrzeugs - eines Tesla Model S - im Jahr 2016 (The Guardian 2016). Dieser weltweit diskutierte Vorfall machte deutlich, dass an die Sicherheit automatisierter Fahrzeuge deutlich andere Maßstäbe angelegt werden. Dabei schneidet die Fahrleistung des im Unfallwagen verwendeten Autopiloten in einer rein statistischen Betrachtung durchschnittlich deutlich besser ab als ein menschlicher Fahrer. Es handelte sich um das erste bekannte Todesopfer bei etwas mehr als 208 Millionen gefahrenen Kilometern insgesamt, bei denen der Autopilot in einem Tesla Model S aktiviert worden war. Im Vergleich dazu: Unter allen Fahrzeugen in den USA gibt es etwa alle 136 Millionen Kilometer (National Highway Traffic Safety Administration 2017) einen Todesfall ${ }^{58}$. Dennoch ist festzuhalten, dass dieser Vorfall für erhebliche Kratzer am Image des automatisierten Fahrens gesorgt hat.

Da die Unfallzahlen der automatisierten Fahrzeuge zumindest in näherer Zukunft deutlich im Fokus einer öffentlichen Debatte stehen werden, geht man davon aus, dass diese gerade mit Hilfe von automatisierten und vernetzten Fahrzeugen um mindestens eine Größenordnung reduziert werden müssen. Nur dann wird die Bevölkerung diese Fahrzeuge als Assistenzsystem akzeptieren (Shashua 2017). Dass dies gelingen kann, zeigt sich daran, dass tatsächliche menschliche Eingriffe in bereits vorhandene automatisierte Fahrfunktionen sich stetig verringern.

\section{Spezielle Hindernisse der Einführung automatisierten Fahrens}

Für die künftige Entwicklung des automatisierten Fahrens insgesamt birgt die gesellschaftliche Wahrnehmung von Unfällen mit dem Fokus auf seltene Einzelfälle und einer fehlenden Relativierung das große Risiko einer nur geringen Akzeptanz von derart für die Sicherheit bedenklichen Anwendungen. Man stelle sich nur einmal die mediale Resonanz auf hypothetische Vorkommnisse vor, wie ein durch Hacking manipuliertes Fahrzeug oder gar eine manipulierte autonome Transportdrohne, die Täter zum Kidnapping oder als Waffe missbrauchen. Neben der Tragik des Einzelfalls würden die davon ausgehenden Schockwellen die Weiterentwicklung automatisier-

Die Aussagekraft des NHTSA-Berichts für den Vergleich der Fähigkeiten menschlicher Fahrer mit denen des Autopiloten wird zum Teil kritisch betrachtet, da der Detailgrad keine Unterscheidung des Anteils der mit aktiviertem Autopilot gefahrenen Kilometern ermöglicht. 
ter Fahrzeuge - die letztendlich ja der Reduzierung der Unfalltoten dienen sollen erheblich zurückwerfen. Das hypothetische Szenario verdeutlicht, wie wichtig ein gut überlegtes Vorgehen bei der Einführung autonomer Fahrzeuge ist.

Der erfolgreiche Einsatz von $\mathrm{KI}$ bei weniger sicherheitsrelevanten Funktionen im Fahrzeug, wie der Fahrer-Fahrzeug-Interaktion (intuitive Sprachsteuerung, Gefühlserkennung, individuelle Fahrermodellierung etc.) hängt ebenfalls stark von der Akzeptanz der Anwender ab. Bei diesen Anwendungen ist mit erheblichen Vorbehalten der Nutzer zu rechnen, wenn die KI etwa zum wiederholten Male die Gefühlslage fehlinterpretiert und das System entsprechend unangemessen auf den Fahrereinwirkt. InAnbetrachtderVielfältigkeitunterschiedlicherFahrerpersönlichkeiten wird es auch für technisch fortgeschrittene Algorithmen eine Herausforderung sein, adäquate und passende Fahrermodellierungen zu generieren. Gerade zu Beginn der Lernphase kann der Nutzen von KI entweder nur fehleranfällig oder wenig individuell sein. Der Verlauf der Lernphase, die Schnelligkeit, mit der eine adäquate Güte der Ergebnisse erzielt wird, und die Akzeptanz der Anwender sowie auch der anderen Verkehrsteilnehmer sind also essenziell, um KI in der Mobilität zu etablieren und letzten Endes das automatisierte Fahren als Ganzes erfolgreich umzusetzen.

\section{KI-Trainingskilometer zur Erhöhung der Sicherheit}

Eine Verbesserung der Zuverlässigkeit der KI in der Fahrzeugführung lässt sich vor allem durch eine größere Menge an Trainingsdaten erreichen. Dabei kommt es neben der Quantität auch auf die Diversität der Daten an, die alle möglichen Szenarien im Straßenverkehr in ausreichendem Maße widerspiegeln müssen. Die vorteilhaftesten Testbedingungen für autonome Fahrzeuge im öffentlichen Verkehrsraum finden sich derzeit in Kalifornien, sodass dort fast jedes Entwicklungsteam Tests durchführt (51 Unternehmen mit mehr als 300 Fahrzeugen und mehr als 1.000 Testfahrern). Aufgrund der Anforderungen der zuständigen Regulierungsbehörde sind diese Testfahrten sowie die Anzahl der darin von den Testfahrern vorgenommenen Deaktivierungen der KI-gestützten Steuerung gut dokumentiert.

Daraus lässt sich einerseits entnehmen, dass sich innerhalb der vergangenen dreieinhalb Jahre die Aktivitäten rapide gesteigert haben (siehe Abbildung 9.3). Andererseits wird deutlich, dass sich mit zunehmender Fahrleistung - insgesamt wurden in Kalifornien mehr als 2,5 Millionen Testkilometer absolviert - ein deutlicher Trend zur Reduktion solcher Fälle abzeichnet, in denen der Testfahrer eingreifen musste. Mehr als 90 Prozent der in Kalifornien gefahrenen Kilometer haben Waymo sowie GM Cruise absolviert. Auch nach der Zulassung für öffentliche Testfahrten in weiteren US-Bundesstaaten wie Arizona und Texas liegt Waymo mit einer Gesamtfahrleistung von knapp 11,3 Millionen Kilometern seit 2009 - allein die Hälfte davon zwischen Juni 2017 und Juni 2018 - vor den Konkurrenten. 
Abschätzungen zeigen jedoch, dass eine Leistungssteigerung der $\mathrm{KI}$ durch eine derartige reine Imitation der menschlichen Fahrweise zu aufwendig und zu kostspielig ist, um Unfallquoten ausreichend zu reduzieren (Zhao und Peng 2017). Doch durch virtuelles Training lässt sich die Diversität sowie das Volumen der Trainingsdaten erhöhen. Beispielsweise ergänzt Waymo die bereits diskutierte Fahrleistung im Realverkehr mit 2,7 Milliarden Simulationskilometern in unterschiedlichen Szenarien. Dazu hat z. B. der Grafikprozessor-Hersteller Nvidia im Januar 2018 das Angebot für Entwickler um die Simulationsumgebung AutoSIM erweitert. Um die Zuverlässigkeit zu gewährleisten, wird derzeit eine begleitete Berechnung von Sicherheitszuständen im Straßenverkehr favorisiert (Shashua 2017), bei denen das Fahrzeug keinen Unfall verursachen kann. Analytische Berechnungen sollen somit eine sichere Fahrstrategie schaffen.

Anzahl der Deaktivierungen der KI-Steuerung pro 1.000 Kilometer (Quartalsdurchschnitt)
Anzahl der Testlizenzen für hochautomatisierte Testfahrten

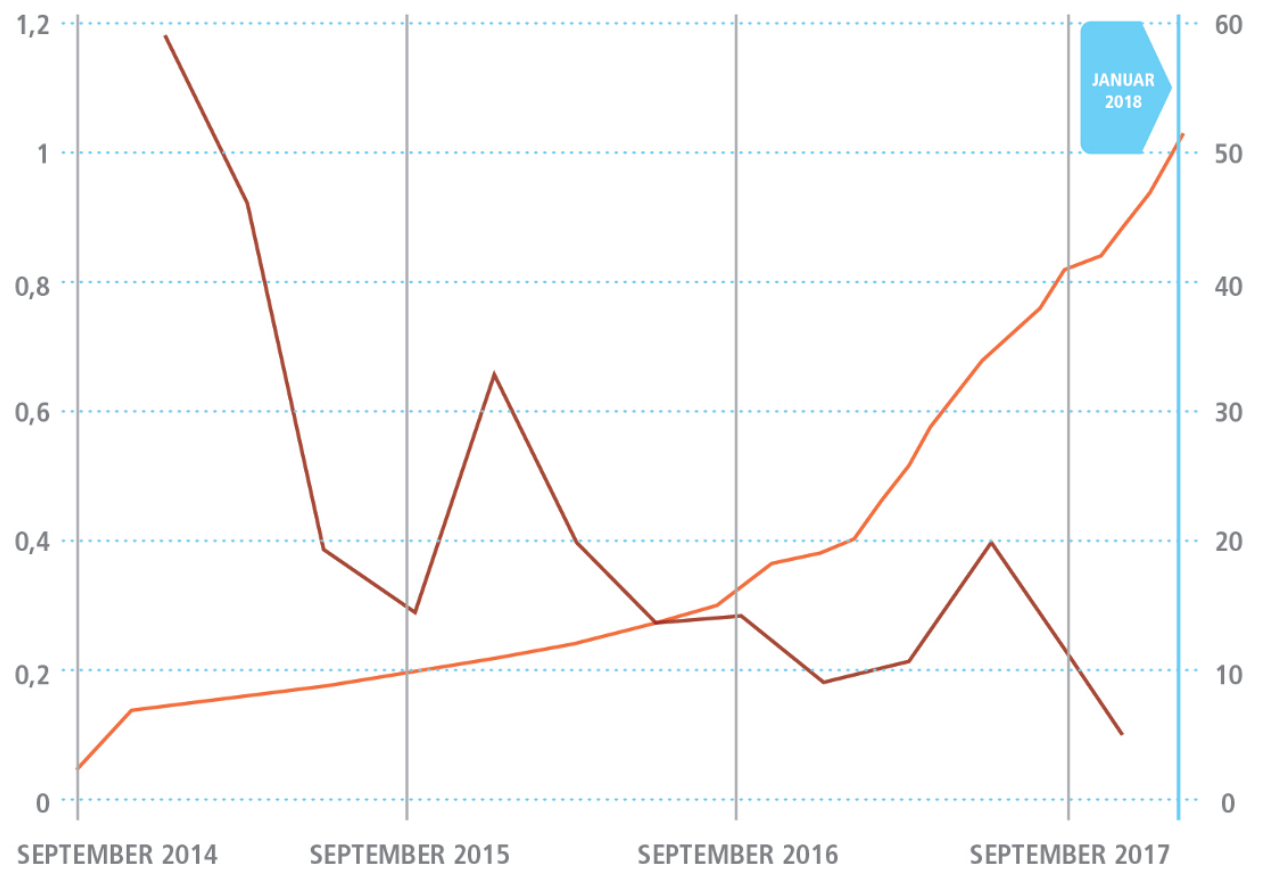

萧茟...

Abbildung 9.3: Anzahl der Deaktivierungen des KI-Steuersystems durch den erforderlichen Testfahrer pro 1.000 Kilometer (rote Linie) und Anzahl der erteilten Testlizenzen für autonomes Fahren im US-Bundesstaat Kalifornien (orangefarbene Linie). 
Seltene, aber gefährliche Verkehrssituationen können anhand von synthetischen Daten in einem für das KI-Training ausreichenden Maße repräsentiert werden. Die virtuelle Umgebung basiert dabei auf Modellen der realen Welt, die allerdings die Frage aufwerfen, welcher Simulationsumfang ausreichend ist, um einen sicheren, der menschlichen Fahrfähigkeit überlegenen, Betrieb zu gewährleisten. Möglichkeiten zur Validierung und Zertifizierung KI-basierter Fahrfunktionen werden daher aktuell sowohl auf nationaler - z. B. beim Genesis-Projekt vom Deutschen Forschungszentrum für Künstliche Intelligenz (DFKI) und TÜV Süd - als auch auf europäischer Ebene - z. B. beim enableS3-Projekt - verfolgt.

\section{Anwendungsbeispiel: Robo-Taxis}

Ein weiteres Argument für die Einführung selbstfahrender Fahrzeuge ist ihr Potenzial, soziale Inklusion zu verbessern. Automatisierte Fahrzeuge können alle Menschen individuell und bedarfsgerecht nutzen. Auch heutige Preisschwellen sind nicht länger ein Hemmnis für die Mobilität des Einzelnen, denn man muss kein Auto mehr kaufen, sondern wie beim Fahrschein im öffentlichen Nahverkehr nur noch eine Mobilitätsdienstleistung bezahlen. Und da für automatisierte Fahrzeuge kein Fahrer erforderlich ist, werden die Kosten für sogenannte Robo-Taxis im Vergleich zu herkömmlichen Taxi- oder Fahrdienstangeboten geringer ausfallen sowie durch $\mathrm{KI}$ geplante bessere Auslastung der Fahrzeuge sowie vorbeugende Wartung weiter sinken. Zusätzliche Ausgaben für die Software und Sensorik können somit kompensiert werden.

Solche Vorteile sprechen für einen Erfolg der Einführung solcher Angebote; einige davon befinden sich bereits im Probebetrieb - z. B. nuTonomy in Boston und Singapur - oder gehen gemäß derzeitiger Planungen bereits bis zum Jahr 2020 oder früher in den praktischen Betrieb über - wie Navya in Paris oder GM Cruise in den USA. Die Marktteilnehmer sind auch in diesem Anwendungsfall sehr heterogen. Neben reinen Mobilitätsdienstleistern wie Uber oder Lyft gesellen sich die Original Equipment Manufacturer (OEM) oder Erstausrüster der klassischen Automobilindustrie wie die Volkswagen AG mit dem Mobilitätsdienstleister MOIA und auch völlig neue Akteure im Straßenverkehr wie ioki von der Deutschen Bahn. Das umfangreichste Angebot stellt dabei derzeit das "Early Rider Program" von Waymo in der US-amerikanischen Stadt Phoenix dar, für das aus mehr als 20.000 Bewerbungen 400 Teilnehmende für eine Probephase ausgewählt wurden, die sich bereits heute jederzeit ein fahrerloses Fahrzeug bestellen und für Alltagsaktivitäten einsetzen können. Die Ambitionen von Waymo lassen sich darüber hinaus durch den Aufbau des Angebots in weiteren US-amerikanischen Städten, z. B. im texanischen Austin, sowie durch die Fahrzeugbestellungen bei Fiat Chrysler in Höhe von 62.000 elektrischen Minivans und bei Jaguar mit 20.000 I-Pace-Geländewagen belegen. Die Herausforderung 
besteht nach Angaben des Unternehmens in der Erweiterung in Gebiete mit komplexeren Wetter- und Straßenbedingungen.

Langfristig gesehen könnte diese Entwicklung die Geschäftsmodelle rund ums Auto grundlegend verändern. Einerseits wird sich der Anteil des Fahrzeugverkaufs am Umsatz verringern, andererseits werden die Einnahmen durch neue Mobilitätsdienstleistungen steigen ("Mobility as a Service“). In einem autonomen Fahrzeug können den Passagieren auch weitere Dienste angeboten werden, da sie vollständig von Aufgaben der Fahrzeugführung entbunden sind. Da sich solche "Shared Mobility"-Angebote besonders für den Transport auf den ersten und letzten Kilometern einer Reise eignen, eröffnet sich hierdurch auch die Möglichkeit einer verkehrsträgerübergreifenden Echtzeit-Routenplanung von Haustür zu Haustür, z. B. Moovel von Mercedes oder die Transportation Mobility Cloud von Ford. Dies wiederum wird ein Anwendungsfeld der KI in Form des maschinellen Lernens sein.

\section{Auswirkungen neuer Mobilitätsangebote}

Die möglichen Auswirkungen der KI-gestützten Mobilitätsdienstleistungen sowie eines neuen persönlichen Mobilitätsverhaltens sind mannigfaltig und lassen sich im Einzelnen nicht präzise vorhersagen. Denkbar ist beispielsweise eine Reduzierung der Anzahl von Kurzstreckenflugreisen zu Gunsten von automatisierten Übernachtfahrten oder eine Gegenbewegung zur Urbanisierung dank der effizienteren Nutzung der Fahrzeit.

Zunächst wird interessant sein zu sehen, wie sich der Stadtverkehr entwickelt. Einerseits wird er durch einen besseren Verkehrsfluss entlastet, andererseits kommt jedoch zusätzlicher Verkehr auf - etwa aufgrund von Bevölkerungswachstum oder vermehrten Logistikfahrten, die bereits heute den Stadtverkehr aufgrund von Online-Bestellungen und anschließenden Einzelauslieferungen belasten. In einem voll- oder hochautomatisierten und mittels Kl optimal koordinierten Verkehrssystem, in dem "Shared Mobility" verbreitet ist und Fahrzeuge im Privatbesitz eine Seltenheit sind, kann man jedoch insgesamt von einer deutlichen Steigerung der Fahrzeugauslastung ausgehen, die bei einem heutigen Stand von unter zehn Prozent (Barter 2013) deutlich verbesserungswürdig ist.

Auch kann aufgrund neuer Mobilitätsangebote der Bedarf an Parkplätzen in den Städten signifikant sinken. Dass sich dies in der Folge positiv auf den Verkehrsfluss auswirken würde, zeigt sich am Beispiel von Berlin, wo derzeit ein Drittel (Cookson und Pishue 2017) der durchschnittlichen Fahrzeit von 22,2 Minuten (Berliner Senatsverwaltung für Umwelt, Verkehr und Klimaschutz 2013) der Parkplatzsuche gilt. Dieses Verkehrsaufkommen würde sich bei einer besseren Fahrzeugauslastung naturgemäß verringern. 
Eine umfassende Vernetzung des hochautomatisierten Transports und die Bereitstellung von Verkehrsinformationen durch die Infrastruktur kann von einer Cloud-basierten KI zur Verkehrsflussoptimierung und zum Flottenmanagement, z. B. der Robo-Taxis, genutzt werden. Die Vernetzung ermöglicht zudem eine optimale Verkehrskoordination, wobei Fahrzeiten sowie Staus minimiert werden. Dies gilt gleichermaßen für die Routenplanung von Lkw-Flotten, für die sich bei uneingeschränkten Betriebszeiten eine höhere Flexibilität ergibt. Auch können die hohen Datenmengen durch eine zentrale KI ausgewertet und für eine vorbeugende Wartung von Fahrzeugflotten verwendet werden.

Außerdem ist die Ausweitung hochautomatisierter Mobilität auch auf den Luftverkehr denkbar. Derzeit erforschen und entwickeln zahlreiche Teilnehmer dieses neuen Markts erste Prototypen (z. B. Airbus, Intel oder Uber) drohnenbasierter Transportmittel. Unabhängig von den zu lösenden technischen Fragen sind auch hier die rechtlichen und sicherheitstechnischen Aspekte sowie die Nutzerakzeptanz sehr hohe Hürden, die eine Einführung auch weit ausgereifter Technologie verhindern können. Denn jeder technische Fehler, der zu einem Absturz führt, kann katastrophale Folgen haben.

\section{Neue Dienstleistungen}

Ein zentrales Element der Dienstleistungen, die die Automobilindustrie den Fahrzeuginsassen selbstfahrender Fahrzeuge künftig anbieten könnte, wäre die Interaktion mit einem persönlichen virtuellen Fahrzeugassistenten, bei dem die KI für Spracherkennung und -verarbeitung sorgt. Da diese Technik in Smartphones und als Teil von Smart Homes schon weit verbreitet ist, würde der Einsatz in Fahrzeugen schlicht eine Erweiterung der Einflusssphären für diese Technik sein und in Gestalt von Smart Cars die Vernetzung vervollständigen. Analog zu den Entwicklungen in den Sektoren Smartphone und Smart Home startet jetzt auch in der Automobilindustrie ein Wettkampf um die Erschließung riesige Mengen personenbezogener Daten. Es sind dieselben Akteure, die sich gegenüberstehen: Die Entwickler der führenden Sprachassistenten Alexa - das sind Toyota, BMW und Ford - sowie von Google - das sind Honda, Hyundai und General Motors, durch den Einsatz in Android Auto, außerdem KIA - haben bereits begonnen, das Feld unter sich aufzuteilen. Sie treffen jedoch auch auf Konkurrenz, die Eigenlösungen anstrebt, dazu zählen Mercedes mit MBUX, Baidu Carlife und Apple mit Siri.

Aufbauend auf den bereits in Smartphones eingesetzten Funktionen kann ein Fahrzeugassistent zusätzlich die biometrische Identifizierung/Authentifizierung der Fahrzeuginsassen, die gestengestützte Anwendungssteuerung sowie eine Fahrer-/Passagierzustandserkennung übernehmen, woraus sich ein weiteres Anwendungsfeld der $\mathrm{KI}$ in der Mobilität ergibt. Den Gefühlszustand sowie die Fahrtüchtigkeit zu erfassen 
spielt beim teilautomatisierten Fahren eine wesentliche Rolle, um Fahrsicherheit zu gewährleisten. Beim vollautomatisierten Fahren bieten sie darüber hinaus Möglichkeiten an, Dienstleistungsangebote weiter zu personalisieren. Beispielsweise plant Toyota aufbauend auf den Assistenten Yui, welcher KI zur Erkennung von Mustern und Routinen verwendet, den Zustand des Menschen festzustellen und diese Information dann zu nutzen, um einen empathischen Assistenten zu schaffen. Denn um das Vertrauen der Fahrzeuginsassen zu gewinnen, muss ein Fahrzeugassistent die Anforderungen eines permanenten Touring-Tests erfüllen. Solche Fahrzeugassistenten könnten sich beispielsweise in Robo-Taxis schnell bezahlt machen, wenn der digitale Assistent auch dazu genutzt wird, das Verhalten der Passagiere zu überwachen, gegebenenfalls Schäden am Fahrzeug einer identifizierten Person zuschreiben und in Rechnung stellen zu können.

Für die Anwendung von KI in der Mobilität konnte innerhalb des zurückliegenden Jahrzehnts die Entwicklung von Hardware und Algorithmen die notwendigen Fortschritte machen. Besonders in den vergangenen Jahren kam es zu einem rasanten Anstieg der Forschungs- und Entwicklungsaktivitäten. Im Fokus steht zumindest in Deutschland das Automobil, da hier mit der Ablösung des Privatfahrzeugbesitzes ein fundamentaler Umbruch bevorsteht. Dieser Wandel bereitet völlig neuen Anwendungen den Weg, und damit einhergehend locken völlig neue Geschäftsmodelle auch neue, überwiegend KI-fokussierte Marktteilnehmer an. Ziel der Entwicklungen ist ein vollständig automatisiertes Verkehrssystem, welches ermöglicht, sowohl die Unfallrate zu reduzieren und die soziale Inklusion der Mobilität zu steigern als auch das Verkehrsmanagement in Verbindung mit einer besseren urbanen Raumplanung zu optimieren. Allerdings entsprechen der Stand der Vernetzung und Digitalisierung von Fahrzeugen und Infrastruktur sowie objektiv unverhältnismäßig hohe Anforderungen an das Sicherheitsniveau noch nicht den Anforderungen eines umfassenden, von der Gesellschaft akzeptierten Einsatzes. Da die Entwicklungsstrategien unterschiedlich und die möglichen Entwicklungspfade mannigfaltig sind, lässt sich zum heutigen Zeitpunkt nur schwer abschätzen, wann hochautomatisiertes Fahren selbstverständlicher Teil des Straßenverkehrs sein und sich das Mobilitätsverhalten aufgrund von individuell wirksamen KI-Anwendungen verändert haben wird.

Vor allem sind zusätzliche disruptive Veränderungen, z. B. die Einführung von Drohnen-Taxis, sowie Entwicklungssprünge im Bereich der KI-Hardware nicht auszuschlieBen. Für eine Prognose lassen sich jedoch als Referenz europäische Roadmaps heranziehen, die hochautomatisiertes Fahren im Jahr 2030 als etabliert betrachten (ERTRAC Arbeitsgruppe Connectivity and Automated Driving 2017). Auf internationaler Ebene kann die als Teil einer sektorübergreifenden KI-Initiative erteilte Vorgabe der chinesischen Regierung gelten, die eine Etablierung von autonomen Fahrzeugen für den Autobahnverkehr innerhalb von drei bis fünf Jahren und für den Stadtverkehr bis 2025 als Richtschnur festschreibt. Auch wenn China bereits über umfangreiche KI- 
Kompetenzen verfügt (Baidu, Tencent usw.), ist diese Richtlinie in Anbetracht der diskutierten notwendigen Voraussetzungen allerdings als sehr ambitioniert einzustufen.

In Anbetracht einer deutlichen Zunahme von Testbetrieben im öffentlichen Verkehr darf man allerdings von einer deutlichen Intensivierung und Beschleunigung der Entwicklungstätigkeiten ausgehen. Zuletzt wurde im Februar 2018 mit der Zulassung von Waymo als Fahrdienstvermittlungsunternehmen im US-Bundesstaat Arizona der Grundstein für eine graduelle Einführung von Robo-Taxidiensten in den Städten gelegt. Nahezu gleichzeitig schuf Kalifornien die rechtliche Grundlage für den Betrieb autonomer Fahrzeuge im öffentlichen Verkehr ab April 2018. Voraussichtlich werden Kl-gestützte Transportangebote bereits in naher Zukunft einen Teil der Alltagsmobilität ausmachen und das Mobilitätsverhalten langfristig grundlegend verändern. 


\section{Literatur}

Barter, Paul (2013): „Cars are parked 95\% of the time". Let's check! Hg. v. Reinventing Parking. Online verfügbar unter https://www.reinventingparking.org/2013/02/cars-areparked-95-of-time-lets-check.html, zuletzt geprüft am 21.02.2018.

Berliner Senatsverwaltung für Umwelt, Verkehr und Klimaschutz (Hrsg.) (2013): Mobilität der Stad. Kenndaten zur Mobilität. Online verfügbar unter https://www.berlin.de/senuvk/ verkehr/politik_planung/zahlen_fakten/download/Mobilitaet_dt_Kap-1-2.pdf, zuletzt geprüft am 21.02.2018.

Cookson, Graham; Pishue, Bob (2017): The Impact of Parking Pain in the US, UK and Germany. Hg. v. INRIX Research. Online verfügbar unter http://inrix.com/research/ parking-pain/, zuletzt geprüft am 21.02.2018.

ERTRAC Arbeitsgruppe Connectivity and Automated Driving (2017): Automated Driving Roadmap.

Ethik-Kommission (2017): Automatisiertes und vernetztes Fahren. Eingesetzt durch den Bundesminister für Verkehr und digitale Infrastruktur.

IEEE Spectrum (2018): Exposing the Power Vampires in Self-Driving Cars. Unter Mitarbeit von Peter Fairley. Online verfügbar unter https://spectrum.ieee.org/cars-that-think/transportation/self-driving/exposing-the-power-vampires-in-self-driving-cars, zuletzt geprüft am 21.02.2018.

National Highway Traffic Safety Administration (NHTSA) (Hrsg.): Automated Vehicles for Safety. Online verfügbar unter https://www.nhtsa.gov/technology-innovation/automatedvehicles-safety, zuletzt geprüft am 21.02.2018.

National Highway Traffic Safety Administration (NHTSA) (2017): 2016 Fatal Motor Vehicle Crashes: Overview. Traffic Safety Facts - Research Note (DOT HS 812 456). Online verfügbar unter https://crashstats.nhtsa.dot.gov/Api/Public/Publication/812456, zuletzt geprüft am 27.02.2018.

Shashua, Amnon (2017): Autonomous Vehicles and Artificial Intelligence: on Achieving a Safe and Scalable Platform. World Knowledge Forum 2017. Seoul, 18.10.2017.

Silver, David; Schrittwieser, Julian; Simonyan, Karen; Antonoglou, loannis; Huang, Aja; Guez, Arthur et al. (2017): Mastering the game of Go without human knowledge. In: Nature 550 (7676), S. 354-359. DOI: 10.1038/nature24270.

Smith, Bryant Walker (2013): Human Error as a Cause of Vehicle Crashes. The Center for Internet and Society (CIS) at Stanford Law School. Online verfügbar unter http://cyberlaw. stanford.edu/blog/2013/12/human-error-cause-vehicle-crashes, zuletzt aktualisiert 2015, zuletzt geprüft am 21.02.2018.

The Guardian (Hrsg.) (2016): Tesla driver dies in first fatal crash while using autopilot mode. Unter Mitarbeit von Danny Yadron und Dan Tynan. San Francisco. Online verfügbar unter https://www.theguardian.com/technology/2016/jun/30/tesla-autopilot-death-self-drivingcar-elon-musk, zuletzt geprüft am 21.02.2018. 
Wu, Ren; Yan, Shengen; Shan, Yi; Dang, Qingqing; Sun, Gang (2015): Deep Image: Scaling up Image Recognition. Hg. v. arXiv. Online verfügbar unter https://arxiv.org/ pdf/1501.02876v3.pdf, zuletzt geprüft am 21.02.2018.

Zhao, Ding; Peng, Huei (2017): From the Lab to the Street: Solving the Challenge of Accelerating Automated Vehicle Testing. Mcity - University of Michigan. Ann Arbor. Online verfügbar unter https://mcity.umich.edu/wp-content/uploads/2017/05/Mcity-White-Paper_ Accelerated-AV-Testing.pdf, zuletzt geprüft am 14.02.2018.

\section{(c) (1)}

Dieses Kapitel wird unter der Creative Commons Namensnennung 4.0 International Lizenz http://creativecommons.org/licenses/by/4.0/deed.de) veröffentlicht, welche die Nutzung, Vervielfältigung, Bearbeitung, Verbreitung und Wiedergabe in jeglichem Medium und Format erlaubt, sofern Sie den/die ursprünglichen Autor(en) und die Quelle ordnungsgemäß nennen, einen Link zur Creative Commons Lizenz beifügen und angeben, ob Änderungen vorgenommen wurden.

Die in diesem Kapitel enthaltenen Bilder und sonstiges Drittmaterial unterliegen ebenfalls der genannten Creative Commons Lizenz, sofern sich aus der Abbildungslegende nichts anderes ergibt. Sofern das betreffende Material nicht unter der genannten Creative Commons Lizenz steht und die betreffende Handlung nicht nach gesetzlichen Vorschriften erlaubt ist, ist für die oben aufgeführten Weiterverwendungen des Materials die Einwilligung des jeweiligen Rechteinhabers einzuholen. 